\title{
Análise biométrica de linhagens de abóbora
}

\author{
Francisco V Bezerra Neto ${ }^{1}$; Nilton R Leal ${ }^{1}$; Fabiane R Costa ${ }^{1}$; Gustavo M Gonçalves'; Antônio T do \\ Amaral Júnior ${ }^{1}$; Hélio O Vasconcellos ${ }^{2}$; Miguel Mello ${ }^{3}$
}

${ }^{1}$ UENF-CCTA-LMGV, Av. Alberto Lamego 2000, Parque Califórnia, 28013-602 Campos dos Goytacazes-RJ; ${ }^{2}$ EMBRAPA/PESAGRORIO, Rod. BR 465, km 7, 23890-000 Seropédica-RJ; E-mail: fvbn@uenf.br

\section{RESUMO}

Cinco caracteres morfoagronômicos, avaliados em sete linhagens de abóbora (Cucurbita moschata), foram submetidos à estimação de parâmetros genéticos e à análise de correlações simples e canônicas. As características foram avaliadas em um experimento em blocos ao acaso, com três repetições e parcelas de seis plantas. Os caracteres peso médio de fruto e comprimento longitudinal externo apresentaram herdabilidade superiores a $80 \%$ e índice de variação superior à unidade, demonstrando que a aplicação de métodos simples de melhoramento como, por exemplo, a seleção massal, poderão resultar em bons ganhos de seleção. Com relação às correlações simples, para a maioria dos pares de características (80\%) as correlações genotípicas foram maiores que as fenotípicas e de ambiente. As correlações simples e canônicas demonstraram que o aumento no peso médio de frutos foi acompanhado de aumento na espessura da polpa e nos comprimentos longitudinal e transversal. Por outro lado, aumento no número médio de frutos apresentou correlação significativa com redução no comprimento longitudinal e na espessura de polpa e com incremento do comprimento transversal. Na população em estudo, genótipos de interesse comercial, contendo fruto de menor tamanho e polpa espessa, poderão ser obtidos com o emprego da técnica do índice de seleção, por sobrepujar o sentido das correlações.

Palavras-chave: Cucurbita moschata, parâmetros genéticos, correlações canônicas, ganhos genéticos, seleção, melhoramento.

\begin{abstract}
Biometric analysis of squash lines

Five morphological and agronomic characteristics were evaluated in seven squash lines. The experiment was carried out in completely randomized blocks, with three replications, and six-plant plots. Genetic parameters, as well as simple and canonic correlations were estimated. Heritability for average fruit weight and external longitudinal length were higher than $80 \%$, with a variation index higher than one. Thus, application of simple breeding methods, such as mass selection, will result in good selection gains. Concerning simple correlations, most of the characteristic pairs $(80 \%)$ had genotypic correlations higher than phenotypic and environmental correlations. Simple and canonical correlations revealed that fruit average weight increased together with increments in pulp thickness and longitudinal and transversal fruit length. On the other hand, plants with more fruits produced smaller fruits (longitudinal length), with thinner pulp, but with higher transversal length. In this population, promising commercial genotypes, producing small fruits with thick pulps, can be obtained using selection indexes.
\end{abstract}

Keywords: Cucurbita moschata, genetic parameters, canonic correlations, genetic gains, selection, breeding.

\section{(Recebido para publicação em 24 de fevereiro de 2005; aceito em 31 de setembro de 2006)}

$\mathrm{A}_{\mathrm{c}}$ nálises biométricas, sobretudo as estimativas de parâmetros genéticos, são de grande importância nos trabalhos de melhoramento. Informações sobre a variância genotípica, herdabilidade e índice de variação são determinantes na escolha do método de melhoramento mais adequado à cultura e permitem fazer inferências sobre a predição de ganhos com a seleção (Cruz \& Regazzi, 2001). Não obstante, o conhecimento das correlações entre características também assume importância relevante, sobretudo quando se deseja obter ganhos indiretos ou mesmo simultâneos em diferentes características. Uma das vantagens desse procedimento, além do ganho em si, é a economia de tempo e de mão-de-obra (Falconer, 1987). A eficiência da seleção de um caráter pode ser aumentada quando se detém este conhecimento, especialmente quando o caráter principal for de difícil seleção a possuir baixa herdabilidade (Cruz \& Regazzi, 2001).

A utilização de análise biométrica via correlações canônicas permite obter gan- hos ainda maiores que a seleção simultânea, uma vez que não se limita à análise de apenas duas características, mas permite a seleção de grupos de características. É possível, por exemplo, confrontar caracteres agronômicos e fisiológicos ou, ainda, agronômicos e caracteres de qualidade de fruto, possuindo assim, uma ampla aplicação no melhoramento de plantas.

Embora à análise biométrica via correlações canônicas seja uma técnica de grande potencial de uso para o melhorista, sua aplicação tem sido rara, vez que nos últimos anos, poucos trabalhos foram desenvolvidos utilizando-se correlações canônicas. Vidigal et al. (1997), por exemplo, trabalhando com mandioca e utilizando as técnicas de correlação simples e canônicas definiram o ideotipo da cultura para a região noroeste do Paraná, com fins industriais. Por sua vez, Amaral Júnior et al. (1997), avaliaram diversas características qualitativas e quantitativas em cinco cultivares de tomate e seus híbridos e, pelo estudo de correlações simples, observa- ram elevada correlação genética entre teor de carotenóides totais, vitamina $\mathrm{C}$ e betacaroteno. Já a análise das correlações canônicas revelou que frutos de maior peso médio tinham maior teor de sólidos solúveis, vitamina $\mathrm{C}$ e betacaroteno. Outrossim, Carvalho et al. (1998) avaliaram a relação existente entre componentes primários e secundários de produção em pimentão através da análise de correlações canônicas e observaram que a associação mais expressiva ocorreu entre caracteres primários de produção (peso médio e número total de frutos) e caracteres de qualidade do fruto (comprimento, largura, relação comprimento/largura e espessura da polpa). Mais recentemente, Viana et al. (2003), por meio de análises de correlações simples e canônicas, determinaram a relação entre caracteres agronômicos e de qualidade de frutos de maracujá em duas populações.

Diferentes trabalhos de pesquisa vêm sendo desenvolvidos com Cucurbitáceas. Porém, para a espécie Cucurbita 
Tabela 1. Estimativas das variâncias fenotípica $\left(\hat{\sigma}_{\mathrm{f}}^{2}\right)$, genotípica $\left(\hat{\sigma}_{\mathrm{g}}^{2}\right)$ e ambiental $\left(\hat{\sigma}^{2}\right)$, da herdabilidade $\left({ }_{h} 2\right)$, dos coeficientes de variação genotípico $\left(C \hat{V}_{g}\right)$ e experimental $\left(C \hat{V}_{\epsilon}\right)$ e do índice de variação ( IV) para características morfoagronômicas de linhagens de abóbora. Campos dos Goytacazes, UENF, 2005.

\begin{tabular}{lrrrrrrc}
\hline Características $^{1}$ & \multicolumn{1}{c}{$\hat{\sigma}_{\mathrm{f}}^{2}$} & \multicolumn{1}{c}{$\hat{\sigma}_{\mathrm{g}}^{2}$} & $\hat{\sigma}^{2}$ & $\mathbf{h}^{2}(\%)$ & $\mathrm{CV}_{\mathrm{g}}$ & $\mathrm{CV}_{\boldsymbol{e}}$ & $\mathrm{IV}$ \\
\hline NMF & 0,19 & 0,13 & 0,06 & 66,07 & 17,10 & 21,22 & 0,80 \\
PMF & 1,66 & 1,42 & 0,24 & 85,63 & 22,97 & 16,29 & 1,41 \\
CLE & 47,68 & 43,86 & 3,81 & 91,99 & 9,22 & 18,04 & 1,96 \\
CTE & 1,46 & 0,94 & 0,52 & 64,40 & 5,74 & 7,40 & 0,77 \\
ESP & 0,11 & 0,08 & 0,03 & 71,16 & 9,33 & 10,29 & 0,91 \\
\hline
\end{tabular}

${ }^{1 / N M F-n u ́ m e r o ~ m e ́ d i o ~ d e ~ f r u t o s ~ p o r ~ p l a n t a ; ~ P M F-p e s o ~ m e ́ d i o ~ d e ~ f r u t o s ; ~ C L E-c o m p r i m e n t o ~}$ longitudinal externo; CTE-comprimento transversal externo; ESP-espessura de polpa.

moschata, os trabalhos de melhoramento ainda são muito incipientes. Embora seja uma hortaliça de expressão no mercado nacional, ocupando o sétimo lugar em volume de produção (Pereira, 1999), cultivares tradicionais, mantidas pelos próprios produtores, ainda são as mais utilizadas para cultivo, vez que são poucas as cultivares desenvolvidas e adaptadas para as regiões produtoras. Pesquisas indicam que o mercado interno prefere frutos menores e com polpa espessa, de onde surge a necessidade de buscar materiais que atendam estas exigências do consumidor.

No final da década de 80 , teve início o programa de melhoramento de abóbora na Empresa de Pesquisa Agropecuária do Rio de Janeiro (PESAGRO-RIO), estação Experimental de Itaguaí. Com o prosseguimento do programa, buscou-se, no presente trabalho, estimar os parâmetros genéticos e quantificar o grau de associação entre componentes primários e caracteres do fruto em linhagens de abóbora, visando gerar informações que permitam selecionar materiais superiores, em parceria com a Universidade Estadual do Norte Fluminense Darcy Ribeiro (UENF).

\section{MATERIAL E MÉTODOS}

Foram utilizadas sete linhagens de abóbora provenientes de cruzamentos entre a cultivar Caravela e um cultigene denominado "São João da Barra". Para a avaliação das características, procedeu-se à implementação de um experimento de campo na unidade de apoio a pesquisa (UAP) da UENF, no ano agrícola de 1999. O delineamento experimental utilizado foi blocos ao acaso, com três repetições e parcelas de seis plantas, dispostas no espaçamento de $3 \mathrm{~m} \times 3 \mathrm{~m}$. O experimento foi conduzido utilizando-se os tratos culturais normal- mente aplicados à cultura.

Foram avaliadas as características número médio de frutos por planta (NMF), peso médio de frutos (PMF), comprimento longitudinal externo (CLE), comprimento transversal externo (CTE) e espessura de polpa (ESP).

Foram estimados os seguintes parâmetros genéticos (Cruz \& Regazzi, 2001): variâncias fenotípica, genotípica e ambiental; herdabilidade; coeficientes de variação genotípica e experimental e índice de variação. As associações entre os pares de características foram obtidas pelas correlações fenotípica, genotípica e ambiental. Para tanto, as covariâncias entre as características X e Y foram calculadas baseando-se no modelo de análise de variância aleatório, tipo II (Vencovsky \& Barriga, 1992; Cruz \& Regazzi, 2001).

Correlações canônicas foram utilizadas para estimar a máxima correlação entre combinações lineares de caracteres distribuídos em dois grupos: (1) componentes primários - NMF e PMF e; (2) caracteres do fruto - CLE, CTE e ESP. Estimaram-se ainda os coeficientes de ponderação dos caracteres em cada combinação linear. Uma correlação canônica é aquela que maximiza a relação entre as combinações lineares $X_{1}$ e $Y_{1}$, sendo $X_{1}=a_{1} x_{1}+a_{2} x_{2}+\ldots+a_{p} x_{p}$; $\mathrm{Y}_{1}=\mathrm{b}_{1} \mathrm{y}_{1}+\mathrm{b}_{2} \mathrm{y}_{2}+\ldots+\mathrm{b}_{\mathrm{q}} \mathrm{y}_{\mathrm{q}},($ Cruz \& Regazzi 2001), sendo estimadas a partir das matrizes:

$\mathrm{R}_{11}=\mathrm{p}$ x q, matriz de correlação entre as características do grupo I;

$\mathrm{R}_{22}=\mathrm{p} \times \mathrm{q}$, matriz de correlação entre as características do grupo II; e

$\mathrm{R}_{12}=\mathrm{p} \times \mathrm{q}$, matriz de correlação entre as características do grupo I e II.

As análises estatísticas foram realizadas com o auxílio do programa computacional Genes (Cruz, 2001).

\section{RESULTADOS E DISCUSSÃO}

Quando os valores da herdabilidade $\left(\mathrm{h}^{2}\right)$ são superiores a $80 \%$ e o índice de variação é superior a unidade, podem ser obtidos ganhos de seleção satisfatórios (Falconer, 1987). Tal situação foi observada para peso médio de fruto e comprimento longitudinal externo (Tabela 1), indicando que genótipos superiores para estes caracteres podem ser obtidos pelo emprego de métodos simples de seleção como, por exemplo, a seleção massal. Todavia, mesmo considerando que na pratica as $\mathrm{h}^{2}$ não são baixas para as demais características, não se exclui a possibilidade de utilização de seleção recorrente genotípica, para obtenção de ganhos superiores, apesar de ser uma estratégia pouco ou ainda não utilizada em abóbora.

Para a maioria dos pares de características (80\%), as correlações genotípicas foram maiores que as fenotípicas e ambientais (Tabela 2), indicando maior influência do componente genético em relação aos demais. Além disso, para $40 \%$ das associações, houve diferença de sinal entre as correlações genotípicas e ambientais, o que pode indicar que as causas de variação genética e ambiental influenciaram as características por meio de mecanismos fisiológicos distintos (Falconer, 1987).

$\mathrm{Na}$ análise de correlação, a presença de colinearidade, decorrente de elevadas estimativas de correlações fenotípicas ou genotípicas entre as características estudadas, produz efeitos diversos nos procedimentos canônicos, tornando os resultados pouco precisos (Carvalho, 1995). Assim, no presente estudo, optou-se por usar apenas os caracteres com ausência de colinearidade.

As correlações positivas elevadas, tanto genotípica quanto fenotípica, entre peso médio de frutos e comprimento longitudinal externo $(0,89$ e 0,87 , respectivamente) e entre peso médio e espessura de polpa $(0,87$ e 0,82 , respectivamente) indicam que genótipos com frutos maiores e de polpa mais espessa poderiam ser selecionados através do peso médio dos frutos, o que facilitaria o processo de seleção, uma vez que o caráter peso médio de frutos é de mais fácil avaliação que os demais. Ao contrário, incrementos na característica número médio de frutos, poderiam representar reduções no peso médio dos frutos, no comprimento longitudinal e também na espessura de polpa, em função das cor- 
relações genotípicas negativas de elevada magnitude entre estes pares de características, e aumento no comprimento transversal dos frutos (Tabela 2).

Em relação às correlações canônicas (Tabela 3), os grupos estudados (grupo 1número médio de frutos por planta e peso médio de frutos; grupo 2- comprimento longitudinal externo, comprimento transversal externo e espessura de polpa) não são independentes, pois apresentaram correlações diferentes de zero pelo teste quiquadrado, a $1 \%$ de probabilidade $(\mathrm{r}=$ $1,30 * *$ e $1,00 * *$, para primeira e segunda correlações canônicas, respectivamente).

Em relação ao primeiro par canônico, é possível concluir que indivíduos com maior número médio de frutos tendem a produzir frutos com menor comprimento longitudinal, menor espessura de polpa e maior comprimento transversal. Portanto, a análise fundamentada nas correlações canônicas (Tabela 3) ratificou o resultado já encontrado nos estudos da correlação simples (Tabela 2). Em relação ao segundo par canônico, verificou-se que maior peso médio de frutos determinou aumento na espessura da polpa e nos comprimentos longitudinal e transversal dos frutos, o que novamente fortalece os resultados já encontrados no estudo das correlações simples (Tabela 2).

Cumpre elucidar que, a despeito das associações simples indicarem a perspectiva de seleção de genótipos não muito interessantes para o mercado consumidor, com base nas correlações genotípicas positivas entre PMF e ESP, a acurada análise das correlações canônicas possibilita uma reflexão mais aprofundada a respeito da perspectiva de sucesso na seleção de genótipos superiores na população em estudo. Isto posto, por uma análise inicial, com fulcro no primeiro par canônico, percebe-se a possibilidade de selecionar frutos de tamanho mediano contendo espessura de polpa menor, face à magnitude das associações entre PMF e ESP de valores respectivos 0,53 e $-0,12$. Todavia, o valor negativo expresso por ESP é próximo de zero, o que, por conseguinte, não invalida a hipótese de seleção de genótipos com fruto de tamanho mediano e com polpa de espessura adequada à preferência do consumidor. Neste aspecto, a experiência do melhorista associada ao uso de procedimentos de análise genético-estatísticas adequadas, como o emprego da estratégia dos índices de seleção, torna possível a seleção de genótipos com menor tamanho de fruto e, concomitantemente, com maior espessura de polpa. O procedimento analítico dos

Tabela 2. Estimativas das correlações fenotípica $\left(r_{f}\right)$, genotípica $\left(r_{g}\right)$ e ambiental $(r)$ entre características morfoagronômicas de linhagens de abóbora. Campos dos Goytacazes, UENF, 2005.

\begin{tabular}{lllllc}
\hline Características $^{1}$ & Correlação & PMF & CLE & CTE & ESP \\
\hline NMF & & $-0,30$ & $-0,41$ & $0,56^{*}$ & $-0,48^{*}$ \\
PMF & & & $0,87^{*}$ & $0,45^{*}$ & $0,82^{*}$ \\
CLE & $r_{f}$ & & 0,29 & $0,53^{*}$ \\
CTE & & & & & 0,19 \\
\hline NMF & & $-0,54^{*}$ & $-0,64^{*}$ & $0,74^{*}$ & $-0,84^{*}$ \\
PMF & & $0,89^{*}$ & 0,39 & $0,87^{*}$ \\
CLE & $r_{g}$ & & & 0,35 & $0,60^{*}$ \\
CTE & & & & & $-0,01$ \\
\hline NMF & & $0,48^{*}$ & $0,52^{*}$ & 0,23 & 0,32 \\
PMF & & $0,72^{*}$ & $0,70^{*}$ & $0,71^{*}$ \\
CLE & $r_{a}$ & & & 0,11 & 0,29 \\
CTE & & & & & $0,61^{*}$ \\
\hline
\end{tabular}

${ }^{*}$ Significativo a 5\% de probabilidade pelo teste t.

${ }^{1 / N M F-n u ́ m e r o ~ m e ́ d i o ~ d e ~ f r u t o s ~ p o r ~ p l a n t a ; ~ P M F-p e s o ~ m e ́ d i o ~ d e ~ f r u t o s ; ~ C L E-c o m p r i m e n t o ~}$ longitudinal

Tabela 3. Estimativas das correlações e pares canônicos entre componentes primários (grupo 1) e caracteres do fruto (grupo 2) em linhagens de abóbora. Campos dos Goytacazes, UENF, 2005.

\begin{tabular}{|c|c|c|}
\hline \multirow{2}{*}{ Componentes primários ${ }^{1}$} & \multicolumn{2}{|c|}{ Coeficientes canônicos } \\
\hline & $1^{\circ} \mathrm{par}$ & $2^{\circ}$ par \\
\hline NMF & 1,18 & 0,13 \\
\hline PMF & 0,53 & 1,06 \\
\hline \multicolumn{3}{|l|}{ Caracteres do fruto ${ }^{1}$} \\
\hline CLE & $-0,67$ & 0,39 \\
\hline CTE & 1,32 & 0,38 \\
\hline ESP & $-0,12$ & 0,58 \\
\hline$r$ & $1,30^{*}$ & $1,00^{*}$ \\
\hline
\end{tabular}

* Significativo a $1 \%$ de probabilidade pelo teste qui-quadrado.

${ }^{1 / N M F-n u ́ m e r o ~ m e ́ d i o ~ d e ~ f r u t o s ~ p o r ~ p l a n t a ; ~ P M F-p e s o ~ m e ́ d i o ~ d e ~ f r u t o s ; ~ C L E-c o m p r i m e n t o ~}$ longitudinal externo; CTE-comprimento transversal externo; ESP-espessura de polpa.

índices de seleção, pela robustez analítica e por sobrepujar o sentido das correlações será, a partir da próxima etapa seletiva do programa, de absoluta relevância na fidedigna seleção dos genótipos de interesse ao mercado consumidor.

\section{REFERÊNCIAS}

AMARAL JÚNIOR AT; CASALI VWD; CRUZ CD; FINGER FL. 1997. Correlações simples e canônicas entre caracteres morfológicos, agronômicos e de qualidade em frutos de tomateiro. Horticultura Brasileira 15: 49-52.

CARVALHO CGP; OLIVEIRA VR; CASALI VWD; CRUZ CD. 1998. Correlações canônicas entre componentes primários e secundários da produção de frutos em pimentão. Horticultura Brasileira 16: 113-118.

CARVALHO SP. 1995. Métodos alternativos de estimação de coeficientes de trilha e índice de seleção sob multicolinearidade. Viçosa: Imprensa universitária, 163p (Tese doutorado).

CRUZ CD. 2001. Programa Genes (Versão Windows): aplicativo computacional em genética e estatística. Viçosa: UFV.
CRUZ CD; REGAZZI AJ. 2001. Modelos biométricos aplicados ao melhoramento genético. 2.ed. rev. Viçosa: UFV. 390p.

FALCONER DS. 1987. Introduction to quantitative genetics. 2. ed. London: Longman. 340p.

PEREIRA W. 1999. Recomendações para a frutificação da abóbora híbrida tipo Tetsukabuto: uso de polinizadores e reguladores de crescimento de plantas. Brasília: Embrapa Hortaliças. 8p. (Comunicado Técnico 12).

VENCOVSKY R; BARRIGA P. 1992. Genética biométrica no fitomelhoramento. Ribeirão Preto: Sociedade Brasileira de Genética. 486p.

VIANAAP; PEREIRA TNS; PEREIRAMG; SOUZA MM; MALDONADO JFM; AMARAL JÚNIOR AT. 2003. Simple and canonic correlation between agronomical and fruit quality traits in yellow passion fruit (Passiflora edulis $f$. flavicarpa) populations. Crop Breeding and Applied Biotechnology 3: 133-140.

VIDIGAL MCG; VIDIGAL FILHO PS; AMARAL JÚNIOR AT; BRACCINI A de LE. 1997. Análise de parâmetros genéticos e correlações simples e canônicas entre características morfoagronômicas e da qualidade das raízes em cultivares de mandioca (Manihot esculenta Crantz) adaptadas ao noroeste paranaense. $R e$ vista Brasileira de Mandioca.15: 41-48. 